\title{
A Survey for Pulsating Ap Stars from Naini Tal
}

\author{
Peter Martinez ${ }^{1}$, D. W. Kurtz ${ }^{2}$, U. S. Chaubey ${ }^{3}$, S. K. Gupta ${ }^{3}$, \\ S. Joshi ${ }^{3}$, R. Sagar ${ }^{3}$, B. N. Ashoka ${ }^{4}$, S. Seetha ${ }^{4}$
}

\section{Pulsation in Am and Ap Stars}

Pulsation is ubiquitous among chemically normal A-type stars, but comparatively rare among chemically peculiar Am and Ap stars of the same temperature range. The conventional explanation for this is that diffusion produces the surface abundance anomalies in the Am and Ap stars, and also drains $\mathrm{He}$ from the $\mathrm{He}-\mathrm{II}$ ionisation zone, thus quenching the $\kappa$-mechanism that drives $\delta$ Scuti pulsation. The pulsating Am and Ap stars exhibit dichotomous pulsation characteristics. The Am stars (and related stars) exhibit low-overtone $\delta$ Scuti pulsation, with amplitudes ranging from a few mmag to $0.1 \mathrm{mag}$. The pulsating Ap stars exhibit high-overtone pulsation with periods in the range 6-16 min and Johnson $B$ semi-amplitudes typically $\leq 5 \mathrm{mmag}$. These stars are referred to as rapidly oscillating Ap stars, or 'roAp' stars (the see review by Martinez \& Kurtz 1995).

In the roAp stars the pulsations are aligned with the magnetic axis, which is inclined to the rotation axis, causing the pulsations to be seen from variable aspect as the star rotates. This gives rise to an observed modulation of the pulsation amplitude and phase with rotation. The model describing the variations of pulsation amplitude and phase is called the oblique pulsator model.

Of the 31 known roAp stars, 28 are in the southern hemisphere and were discovered mostly at the Sutherland site of the South African Astronomical Observatory, where excellent photometric conditions are common. To stimulate more work on roAp stars, we have initiated a northern-sky survey of the roAp phenomenon, based on experience gained in the southern survey.

Naini Tal was selected as the site for our survey because of its northern location and the excellent photometric conditions that prevail at certain times of the year. The observatory is situated on Manora Peak at longitude $79^{\circ} 27^{\prime} \mathrm{E}$, latitude $29^{\circ} 22^{\prime} \mathrm{N}$ and altitude $1951 \mathrm{~m}$. The best observing period is from October to April.

\section{Candidate Selection and Observations}

Candidates for our survey are drawn from the catalogue of CP stars of Renson, Gerbaldi, \& Catalano (1991), and from the Simbad database. We concentrate

\footnotetext{
${ }^{1}$ SAAO, P.O. Box 9, Observatory 7935 , South Africa

${ }^{2}$ Department of Astronomy, University of Cape Town, Rondebosch 7701, Sonth Africa

${ }^{3}$ Uttar Pradesh State Observatory, Manora Peak, Naini Tal 263 129, India

${ }^{4}$ Technical Physics Division, ISRO Satellite Centre, Vimanapura PO, Airport Rd, Bangalore 560017 , India
} 
on stars with Strömgren photometric indices similar to those determined for the known roAp stars: $0.08 \leq b-y \leq 0.29,0.20 \leq m_{1} \leq 0.32, \delta m_{1} \geq 0.01$, $0.49 \leq c_{1} \leq 0.85, \delta c_{1} \leq 0.04$, and $2.70 \leq \beta \leq 2.83$. Indices in these ranges are not an unambiguous indicator of roAp pulsation. Nor do they separate Am and Ap stars, a distinction which can only reliably be made on spectroscopic grounds. Nonetheless, these indices serve to narrow down the field of candidates to the most promising subset.

The observations are acquired using a high-speed photometer attached to the 1-m Sampuranand telescope of the Uttar Pradesh State Observatory at Naini Tal. Candidate stars are observed in Johnson $B$ light with continuous 10-s integrations. The observations are interrupted for occasional measurements of the sky background.

The detection limits for the roAp oscillations are defined by sky transparency variations at low frequencies, and by the level of the scintillation noise for higher frequencies. Because roAp star oscillations are modulated by both beating among modes and projection effects from oblique pulsation, candidates need to be observed several times to establish a null result for pulsation.

\section{First Results}

To date, high-speed photometric observations of 27 candidate stars have been acquired. Data are inspected for indications of low-frequency variability before being prewhitened to search for roAp oscillations.

Since the photometric criteria used to select candidates do not separate $\mathrm{Am}$ and Ap stars, a by-product of this survey is the discovery of pulsations in some Am stars which have been mis-classified as Ap stars. We believe that the first two pulsators discovered in this survey are probably Am stars. HD 13038 (Martinez et al. 1999a) pulsates with periods of 28 and 34 minutes and a $B$ peak-to-peak amplitude of $3 \mathrm{mmag}$. This star has a Draper classification of A3 and has Strömgren colours similar to the Ap and Am stars. HD 13079 pulsates with a period of $72 \mathrm{~min}$ and is a probable Am fundamental-mode pulsator on the red edge of the instability strip (Martinez et al. 1999b). Oscillations with such long periods can easily be masked by sky transparency variations. This illustrates the photometric quality of Naini Tal and the importance of careful examination of the nature of low-frequency 'noise' in the data.

\section{References}

Martinez, P. \& Kurtz, D. W. 1995, Ap\&SS, 230, 29

Martinez, P., Ashoka, B. N., Kurtz, D. W., Gupta, S. K., \& Chaubey, U. S. 1999a, Inf. Bull. Var. Stars, No. 4677

Martinez, P., Kurtz, D. W., Ashoka, B. N., Chaubey, U. S., Gupta, S. K., Leone, F., Catanzaro, G., Sagar, R., Raj, E., Seetha, S., \& Kasturirangan, K. 1999b, MNRAS, 309, 871

Renson, P., Gerbaldi, M., \& Catalano, F. A. 1991, A\&AS, 89, 429 